\title{
Vector Competence of Aedes albopictus Populations from the Northeastern United States for Chikungunya, Dengue, and Zika Viruses
}

\author{
Andrea Gloria-Soria, ${ }^{1 *}$ Anne F. Payne, ${ }^{2}$ Sean M. Bialosuknia, ${ }^{2}$ Jessica Stout, ${ }^{2}$ Nicholas Mathias, ${ }^{2}$ Gillian Eastwood, ${ }^{1,3}$ \\ Alexander T. Ciota, ${ }^{2,4}$ Laura D. Kramer, ${ }^{2,4}$ and Philip M. Armstrong ${ }^{1}$ \\ ${ }^{1}$ Department of Environmental Sciences, Center for Vector Biology and Zoonotic Diseases, The Connecticut Agricultural Experiment Station, New \\ Haven, Connecticut; ${ }^{2}$ The Arbovirus Laboratories, Wadsworth Center, New York State Department of Health, Slingerlands, New York; ${ }^{3}$ Department \\ of Entomology, Virginia Polytechnic Institute and State University, Blacksburg, Virginia; ${ }^{4}$ Department of Biomedical Sciences, State University of \\ New York at Albany School of Public Health, Albany, New York
}

\begin{abstract}
The Asian tiger mosquito (Aedes albopictus) is an important vector of a number of arboviruses, including Zika (ZIKV), dengue (DENV), and chikungunya (CHIKV) viruses, and has recently expanded its range in the eastern United States to southern New England and New York. Given the recent establishment and proliferation of Ae. albopictus in this region and the increasing amount of international travel between the United States and endemic countries, there is a need to elucidate the public health risk posed by this mosquito species in the Northeast. Accordingly, we evaluated the competence of four Ae. albopictus populations from Connecticut and New York, for two strains each of ZIKV, DENV serotype 2 (DENV-2), and CHIKV, currently circulating in the Americas, to evaluate the local transmission risk by this vector. We found that local Ae. albopictus populations are susceptible to infection by all three viruses but are most capable of transmitting CHIKV. Variation in competence was observed for ZIKV and CHIKV, driven by the virus strains and mosquito population, whereas competence was more homogeneous for the DENV-2 strains under evaluation. These results suggest that under optimal circumstances, Ae. albopictus could support localized transmission of these viruses and emphasize the importance of maintaining mosquito surveillance and control programs to suppress Ae. albopictus populations and limit further range expansion of this species.
\end{abstract}

\section{INTRODUCTION}

The Asian tiger mosquito (Aedes albopictus) is an important arbovirus vector and one of the most invasive insect species in the world. During the last 40 years, this species has expanded its range from East Asia to more than 50 countries in Europe, Asia, Africa, and the Americas. ${ }^{1}$ Aedes albopictus was first discovered in the United States in the 1980s, in Memphis, TN, and Houston, TX. Since then, it has spread throughout much of the eastern United States, with established populations documented as far north as southern New England and New York. $^{2-4}$ Aedes albopictus thrives in urban and suburban habitats where it readily feeds on people, making it an important pest species. ${ }^{5,6}$ It is also a competent vector of more than 20 arboviruses in the laboratory and serves as an important vector of dengue virus (DENV) and chikungunya virus (CHIKV), in areas where the main vector (Aedes aegypti) is absent or rare. ${ }^{7,8}$ Aedes albopictus has played a primary role in driving epidemics of CHIKV in the Indian Ocean Islands (2004-2007) and Italy (2007 and 2017), and of DENV in the Hawaiian Islands (2001, 2011, and 2015), China (2009), and Japan (2014). ${ }^{9-15}$

The number of human infections of Zika virus (ZIKV), DENV, and CHIKV has increased in their endemic ranges and expanded to new geographic regions. ${ }^{16,17}$ These trends are driven, in part, by increased trade and travel and the expansion and proliferation of Aedes vectors. ${ }^{18}$ Given the recent expansion of Ae. albopictus in the Northeastern United States and the presence of major urban centers that serve as frequent entry points for travelers from endemic countries, there is an

\footnotetext{
*Address correspondence to Andrea Gloria-Soria, Department of Environmental Sciences, Center for Vector Biology and Zoonotic Diseases, The Connecticut Agricultural Experiment Station, 123 Huntington St., P.O. Box 1106, New Haven, CT 06504. E-mail: andrea.gloria-soria@ct.gov
}

increasing threat from these arboviruses in the region. ${ }^{19,20}$ Zika virus, DENV, and CHIKV are repeatedly imported to the United States by travelers, and one locally acquired case of DENV has been reported from Long Island, NY, in 2013 (CDC ArboNet; www.cdc.gov). Previous studies indicate that vector competence is population- and virus strain-dependent, and that $A$ e. albopictus populations from NY can transmit ZIKV. ${ }^{21,22}$ Nevertheless, the competence of local populations of Ae. albopictus for other potentially invasive arboviruses remains unknown.

In this study, we compared the vector competence of $\mathrm{Ae}$. albopictus populations from Connecticut (CT) and New York (NY) for low-passage strains of ZIKV, DENV serotype 2, and $\mathrm{CHIKV}$, to evaluate the susceptibility and ability of local populations to transmit these viruses, and thus better understand the risk posed by this mosquito species. Mosquitoes were exposed to virus-infected blood meals and harvested at various time points to evaluate the percentage of mosquitoes that acquire infection and subsequently transmit virus. In addition, a laboratory colony of $A$ e. aegypti was included to monitor for experimental variation between laboratories involved in this study.

\section{MATERIALS AND METHODS}

Mosquito populations. Aedes albopictus eggs were collected using oviposition traps in Bridgeport (CT), Branford $(\mathrm{CT})$, Fire Island (NY), and Spring Valley (NY), during the summer of 2018. Colonies were subsequently generated at the Connecticut Agricultural Experiment Station (CAES), CT or the Wadsworth Center, NY by blood-feeding adults defibrinated sheep blood (HemoStat Labs, Dixon, CA) on an artificial membrane system and providing egg-laying substrates. Larvae were reared from eggs in plastic trays and fed with either TetraMin ${ }^{\circledR}$ tropical flakes (Tetra GMBH, Melle, Germany) or Kaytee Koi's Choice Premium Fish Food (Kaytee Products Inc., Chilton, WI). Adults were kept in $30 \times 30 \times 30 \mathrm{~cm}$ 
cages and provided with a $10 \%$ sucrose solution ad libitum on a cotton ball. Adult mosquitoes from laboratory generations 2-6 (CT) and 6-9 (NY) were used in this study. Adults from the CAES Orlando strain (ORL) of Aedes aegypti ${ }^{23}$ were used as a control at both laboratories.

Arbovirus strains. Two strains of Zika, dengue serotype 2, and CHIKV were used to challenge adult mosquitoes (Table 1). Zika virus HND C3 (2016-19563, GenBank accession no. KX906952) was isolated in the NYS Arbovirus Laboratory from the serum of a patient who had traveled to Honduras in early 2016 and was passaged one time on Vero cell culture and two times on C6/36 cell culture. Zika virus PRVABC59 (kindly provided by the CDC, Fort Collins, CO; GenBank accession no. KU5012 15) was initially obtained from the serum of a patient who had traveled to Puerto Rico in 2015 and was passaged three times on Vero cell culture and one time on C6/36 cell culture. DENV serotype 2 (DENV-2) NI-2B strain 306 (PRJNA32415.306.1, BioProject PRJNA31235) was isolated from the serum of a patient in Nicaragua in 2007 and passaged three times on C6/36 cell culture (provided by E. Harris, UC Berkley). DENV-2 125270 (GenBank accession no. U91870.1) was originally isolated from the serum of a patient in Venezuela in 1993 and passaged twice on C6/36 cell culture. Chikungunya virus IDR140025461 was isolated in the NYS Arbovirus Laboratory from the serum of a patient in the Dominican Republic in 2014 and passaged once on Vero cell culture and once on C6/36 cell culture. Chikungunya virus LR2006OPY1 (GenBank accession no. KT449801.1) was obtained from the World Reference Center for Arboviruses at the University of Texas Medical Branch, Galveston, TX. This strain was originally isolated from serum of a patient returning from La Réunion Island in 2006, passed five times on Vero cell culture, once in suckling mice, and once in $\mathrm{C6} / 36$ cells. Each virus stock was titrated by plaque assay on Vero cells as previously described. ${ }^{24}$ All aliquots from each of the viral strains originated from the same cell culture and were immediately frozen. Aliquots were only thawed once on the day of the experiment to minimize the effect of virus aliquot on the experimental outcome. Infectious blood meals were prepared from frozen aliquots for CHIKV, whereas fresh virus had to be used for ZIKV and DENV-2 due to problems with the infectivity of frozen stocks (pilot study and Ciota et al. ${ }^{22}$ ). In brief, confluent cell monolayers of $\mathrm{C} 6 / 36$ cells (American Type Culture Collection \#CRL-1660) grown in Dulbecco's modified Eagle's minimum essential medium (Gibco) supplemented with $10 \%$ fetal bovine serum (FBS) and 1\% penicillin and streptomycin were infected with $100 \mu \mathrm{L}$ of a titrated stock virus and incubated at room temperature for 1 hour, rocking every 15 minutes to promote viral attachment. Subsequently, $5 \mathrm{~mL}$ of fresh media were added, and cultures were incubated for 5 days at $28^{\circ} \mathrm{C}$ under a $95 \%$ relative humidity and $5 \% \mathrm{CO}_{2}$ atmosphere. Fresh virus was diluted 10-fold into virus media (fresh media minus FBS), and blood meals were prepared 1:1 with defibrinated sheep blood. Aliquots of the infectious blood meal were frozen for later titration by plaque assay as described in Payne et al. ${ }^{24}$

Mosquito viral challenges. Batches of $\sim 120$ adult female mosquitoes (6-12 days old) were simultaneously challenged with one of each of the two viral strains. Experiments for each virus were performed independently. Connecticut populations were challenged as two (CHIKV and DENV-2) or three (ZIKV) independent replicates. The replicates were performed on different days, with new batches of mosquitoes, and new virus aliquots. Only one replicate was performed with the NY populations, because the overall number of successful individual mosquito challenges per treatment was higher than that in CT. In brief, 24-hour sugar-deprived adult females were offered an infected blood meal (Table 1) using an artificial membrane system lined with hog sausage casing. After 45 minutes, visually engorged females were sorted on ice and incubated for 7 and 14 days post-feeding following holding at a 15:9 hour $\mathrm{L}: \mathrm{D}$ cycle with a temperature cycle of $27: 22^{\circ} \mathrm{C}$, respectively. These conditions were used to approximate mean day- and night-time temperatures and day length in New York City during the month of July. Because dissemination of CHIKV virus has been reported to have a shorter extrinsic incubation period than that of DENV2 and ZIKV, an additional time point at day 4 post-feeding was included for this virus. ${ }^{25-29}$ A $10 \%$ sugar solution was provided on fresh cotton balls ad libitum during the time of incubation. At the end of the incubation period, mosquitoes were anesthetized on ice, their legs and wings removed, and saliva was collected by inserting their proboscis into $20 \mu \mathrm{L}$ pipet tips containing $5 \mu \mathrm{L}$ of a $50 \%$ sucrose $1: 1$ solution in FBS for $\sim 45$ minutes. The content of the pipet tip was then expelled into $50 \mu \mathrm{L}$ of PBS-G (phosphate-buffered saline, $30 \%$ heat-inactivated rabbit serum, and $0.5 \%$ gelatin). Bodies and legs were homogenized in $200 \mu \mathrm{L}$ of PBS-G using Copperhead copper beads (Crosman Corporation, Bloomfield, NY) and a Mixer Mill 400 (Retsch) for 30-60 seconds at 24 $\mathrm{hz}$. All samples were stored at $-80^{\circ} \mathrm{C}$ until RNA extraction.

Despite our efforts to replicate the methods across laboratories, a few methodological differences between CAES (CT) and the Wadsworth Center (NY) were noted during the analysis stage. These include 1) not dissecting the wings of the mosquito for salivation; 2) the use of capillary tubes filled with the $50 \%$ sucrose solution expelled into $150 \mu \mathrm{L}$ of mosquito diluent (MD) (phosphate-buffered saline containing 20\% heat-inactivated FBS plus $50 \mu \mathrm{g} / \mathrm{mL}$ penicillin/streptomycin, $50 \mu \mathrm{g} / \mathrm{mL}$ gentamicin, and $2.5 \mu \mathrm{g} / \mathrm{mL}$ fungizone), rather than using $20 \mu \mathrm{L}$ pipet tips containing $5 \mu \mathrm{L}$ of the solution expelled into $50 \mu \mathrm{L}$ of PGS-G; 3) homogenization of bodies in $500 \mu \mathrm{L}$ of MD instead of $200 \mu \mathrm{L}$ of PBS-G buffer; and 4) 5-mm zinc-plated BBs (Daisy Rogers, AR) rather than copper beads.

TABLE 1

Virus strains used to measure competence on Aedes albopictus populations from Connecticut and New York

\begin{tabular}{lllcc}
\hline Virus & \multicolumn{1}{c}{ Strain } & Geographic origin & Year of isolation & Blood meal titer (log10 PFU/mL) \\
\hline ZIKV & PRVABC59 & Puerto Rico & 2015 & $7.3-7.9$ \\
ZIKV & HND C3 & Honduras & 2016 & $7.4-7.8$ \\
DENV-2 & NI-2B strain 306 & Nicaragua & 2007 & $7.6-8.3$ \\
DENV-2 & 1252620 & Venezuela & 1993 & $7.5-7.8$ \\
CHIKV & IDR140025461 & Dominican Republic & 2014 & 7.4 \\
CHIKV & LR2006OPY1 & La Reunion Island & 2006 & 7.4 \\
\hline
\end{tabular}

CHIKV = Chikungunya virus; DENV-2 = Dengue serotype 2 virus; ZIKV = Zika virus. 
Virus detection. Viral RNA was extracted at CAES from 50 $\mu \mathrm{L}$ of the frozen samples (homogenized bodies, legs, and saliva) using the KingFisher ${ }^{\mathrm{TM}}$ Flex Purification System (ThermoFisher Scientific, Waltham, MA) with the Mag-Bind ${ }^{\circledR}$ Viral DNA/RNA Kit (Omega Bio-Tek, Norcross, GA) and eluted in a final volume of $50 \mu \mathrm{L}$ of $1 / 10 X$ TE buffer (Tris-EDTA). Two microliters of this eluate were then used for each $10 \mu \mathrm{L}$ realtime PCR (RT-PCR) reaction. The presence of virus was determined in duplicate via RT-PCR with the I-Taq ${ }^{\mathrm{TM}}$ Universal probes 1-step-kit (BioRad Laboratories, Hercules, CA), using primers and probes described in Supplemental Table S1. Samples were only thawed once. A mosquito was considered positive for infection if viral RNA was detected in the body (cycle threshold $[\mathrm{Ct}]<35$ ), the infection was considered disseminated if viral RNA was detected in legs ( $\mathrm{Ct}<37$ ), and the mosquito was considered able to transmit if viral RNA in saliva was detected $(\mathrm{Ct}<38)$. Reported rates are the percentage of mosquitoes tested that were positive at a specific tissue.

New York samples were initially tested by plaque assay on Vero cells and subsequently confirmed by RT-PCR. Viral RNA was extracted from $50 \mu \mathrm{L}$ of the samples (homogenized bodies, legs, and saliva) using the Applied Biosystems MagMAXTMExpress 96 extractor (ThermoFisher Scientific, Waltham, MA) using MagMAX-96 Viral RNA Isolation Kit Cat\# AM1836 (Ambion, Austin, TX.) and eluted in a final volume of $90 \mu \mathrm{L}$ of sterile millipore $\mathrm{H}_{2} \mathrm{O}$. Five microliters of this elution were then used for each $15 \mu \mathrm{LRT}$-PCR reaction. The presence of virus was determined by RT-PCR with qScript ${ }^{\mathrm{TM}}$ XLT 1-step RT-PCR ToughMix, Low ROX (Quanta biosystems, Beverly, $\mathrm{MA}), 10 \mu \mathrm{L}$ of $100 \mu \mathrm{M}$ primer stock, and $8 \mu \mathrm{L} 45 \mu \mathrm{M}$ stock of probe in a total of $1,040 \mu \mathrm{L}$ of master mix. Samples were considered positive if the Ct value fell above the lowest detectable pfu value as determined by the standard curve for each individual assay (approximately $1 \mathrm{pfu} / \mathrm{sample)}$ ). It is important to note that despite using larger elution volumes relative to the CT protocol, the standard curve indicates that the assay sensitivity is sufficient to identify positive samples with low viral loads. These cutoff Ct values were consistent with CAES cutoffs.

The number of mosquitoes tested by RT-PCR and those recorded as positive can be found in Supplemental Table S2.

Statistical analysis. Results from the virus challenges were analyzed by considering the presence of the virus as a binary response (positive/negative) in a full-factorial generalized linear mixed model that included laboratory (when applicable), mosquito population, virus strain, and days postinfection (dpi) as predictors, as well as their interactions, incorporating replicates as a random effect (CT only). The model was fitted as binomial with a logit link function. Analysis of dissemination was based on samples positive for infection. Likewise, analysis of transmission was based on samples positive for dissemination. Statistical significance was assessed via analysis of variance using a type II Wald chi-square test at a threshold of $P=0.05$. All analyses were implemented using the stats package from the $\mathrm{R}$ software, ${ }^{30}$ and the $/ m e 4^{31}$ and $c a r^{32}$ packages.

\section{RESULTS}

Zika virus. Aedes albopictus from CT and NY were challenged with two strains of ZIKV (HND C3 [HND] and PRVABC59 [PRV]; Table 1), to determine whether mosquito populations vary in competence. Mosquitoes were harvested on days 7 and 14 postinfection (dpi) to determine the percentage of mosquitoes infected with the virus (bodies), with disseminated infections (legs), and capable to transmit the virus (saliva secretions). Aedes aegypti (ORL strain) were assayed in parallel at $14 \mathrm{dpi}$ to monitor experimental variation between the laboratories in CT and NY, where vector competence trials were conducted. Infection rates in the $\mathrm{ORL}$ strain ranged from 42 to $60 \%$ and were not significantly different between laboratories (Supplemental Table S2). By contrast, the dissemination rates varied from $7 \%$ to $60 \%$ and were consistently higher at the NY laboratory $(P<0.001$; Supplemental Table S3). Given these differences, we analyzed the results of the experiments from CT and NY separately for all subsequent comparisons.

Overall, populations of Ae. albopictus were highly susceptible to infection by both strains of ZIKV (63-98\%; Figure 1). Mosquitoes readily developed disseminated infection by day 14 (15-98\%), but very few had detectable virus in saliva (Figure 1, Supplemental Table S2). Analysis of the Ae. albopictus data from $\mathrm{CT}$ revealed no significant differences in virus infection and dissemination among virus strains and mosquito populations. Virus dissemination was observed only at $14 \mathrm{dpi}$ in CT populations (Figure 1, Supplemental Tables S2 and S4). By contrast, Ae. albopictus populations from NY exhibited more variation in virus infection and dissemination rates, with evidence of dissemination starting at day $7 \mathrm{dpi}$ (Figure 1). Virus strain and mosquito population, as well as their interaction, contributed to differences in the infection rate in NY $(P \leq 001$; Supplemental Table S5). In these samples, higher infection rates were detected with the PRV strain of ZIKV than with HND, with $90 \%$ and $98 \%$ versus $63 \%$ and $98 \%$, for Fire Island and Spring Valley, respectively, at $14 \mathrm{dpi}$ (Figure 1, Supplemental Table S2). Viral dissemination increased with the time elapsed after infection (dpi), and was also influenced by mosquito population and its interaction with dpi $(P \leq 0.009$, Supplemental Table S5). Overall, mosquitoes from Spring Valley were more susceptible than those from Fire Island to both viruses and showed higher dissemination rates. Because of the low number of positive saliva samples, analysis of transmission rate was not performed (Supplemental Table S2). However, Spring Valley was the only Ae. albopictus population with positive saliva samples ( $N=1$ at each dpi 7 and 14).

Dengue virus. Aedes albopictus competence for DENV-2 was assayed by exposing the CT and NY populations to two strains of the virus ( $\mathrm{NI}-2 \mathrm{~B}$ and 1252670; Table 1). Following the same protocol as described earlier, mosquitoes were harvested on days 7 and 14 postinfection (dpi), and the percentage of mosquitoes infected, with a disseminated infection, and those capable of transmission, was estimated (Figure 2, Supplemental Table S2). Aedes aegypti (ORL strain) was infected in parallel and sampled at $14 \mathrm{dpi}$ as a control for variation of DENV-2 infections between laboratories. Analysis of the ORL control found no difference between laboratories in infection or dissemination rates (Supplemental Table S3), with infection ranging from $90 \%$ to $98 \%$ and dissemination from $76 \%$ to $85 \%$. Despite these results, and based on the differences observed with the other viruses in this study, we followed a conservative approach and analyzed the data from each laboratory separately.

Aedes albopictus populations were highly susceptible to DENV-2 infection (> 93\% of mosquitoes) (Figure 2 and Supplemental Table S2). More than half of the mosquitoes exposed to DENV-2 developed disseminated infections 

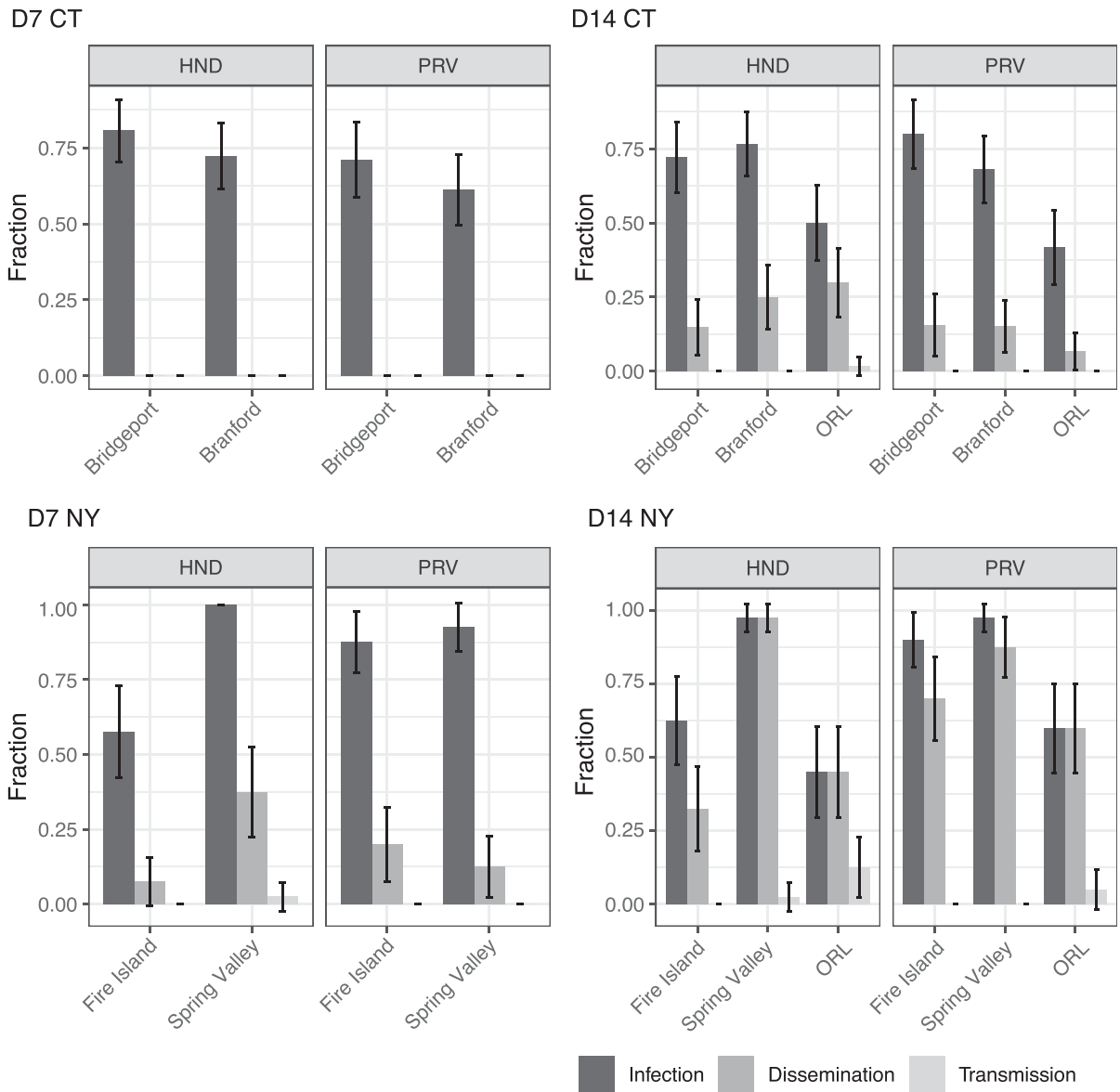

FIGURE 1. Zika virus infection, dissemination, and transmission rates of Aedes albopictus populations from Connecticut (CT: Bridgeport and Branford) and New York (NY: Fire Island and Spring Valley) at days 7 (D7) and 14 pi (D14). Percentages shown are based on the total number of mosquitoes tested. The virus strain used for the challenge is indicated in the top of each panel (HND: HND C3, PRV: PRVABC59). Error bars represent $95 \% \mathrm{Cl}$. Aedes aegypti (ORL) control is shown.

(49-83\%) by day 14 , but relatively few had detectable virus in the saliva (0-5\%). Further analysis indicates that population, virus strain, not dpi influenced the infection rate (Supplemental Tables S4 and S5). Dissemination rate increased with dpi $(P<$ 0.001; Supplemental Tables S4 and S5; Figure 2) and was homogeneous across the populations. Positive saliva was exclusively detected for the $\mathrm{NI}-2 \mathrm{~B}$ strain, but statistical analysis was prevented by the small sample size (Figure 2; Supplemental Table S2).

Chikungunya virus. Competence for CHIKV was assayed by exposing populations of Ae. albopictus from CT and NY to strains IDR140025461 [IDR] and LR2006OPY1 [OPY1] (Table 1), as described earlier. Mosquitoes were harvested on days 4, 7, and 14 postinfection (dpi), and the percentage of mosquitoes infected, with a disseminated infection, and those capable of transmission, was estimated (Figure 3, Supplemental Table S2). Aedes aegypti (ORL strain) was infected in parallel and sampled at $14 \mathrm{dpi}$ to control for variability in CHIKV infections across the laboratories involved in the study. Infection rates in Ae. aegypti were higher in NY relative to CT (CT: $92 \%$ and $97 \%$ versus NY: $100 \% ; P=0.0154$, Supplemental Table S3). Dissemination rates were also higher between laboratories, but the difference was not statistically supported (CT: $89-92 \%$ versus NY: 95-100\%; Supplemental Table S3). Given these results, the data from each laboratory were analyzed independently.
Aedes albopictus was highly susceptible to infection by $\mathrm{CHIKV}$, with infections achieving high numbers beginning at 4 dpi (> 70\%), (Figure 3, Supplemental Table S2). Dissemination rates varied with $\mathrm{dpi}$, the virus infecting, and the mosquito population, ranging from $30 \%$ to $100 \%$ after $14 \mathrm{dpi}(P \leq 0.006$, Supplemental Tables S4 and S5; Figure 3). The OPY1 strain of $\mathrm{CHIKV}$ led to significantly more disseminated infections at every time point. High dissemination rates were observed in NY starting at day 7 pi (> 80\%), with Fire Island consistently showing lower dissemination rates relative to Spring Valley, NY $(P<0.001$; Supplemental Tables S2 and S5, and Figure 3). Dissemination rates in CT were considerably lower than those in NY, ranging from $30 \%$ to $37 \%$ (IDR) and $65 \%$ to $60 \%$ (OPY1) at $14 \mathrm{dpi}$; see Figure 3. Lower dissemination rates were observed in Branford than in Bridgeport with strain OPY1, only at 7 dpi $(P<0.001$, Supplemental Tables S2 and S4, and Figure 3).

Chikungunya virus was detected in saliva from infected mosquitoes as early as $4 \mathrm{dpi}$, with transmission rates across the experiment ranging from $0 \%$ to $28 \%$ (Figure 3 and Supplemental Table S2). The higher number of positive samples was recorded at $7 \mathrm{dpi}$ (CT: $P=0.0441$, Supplemental Table S4; NY: $P=0.033$, Supplemental Tables S2 and S5 and Figure 3). Mosquitoes infected with the OPY1 strain were more likely to show virus in saliva (CT: $P=0.0079$, Supplemental 

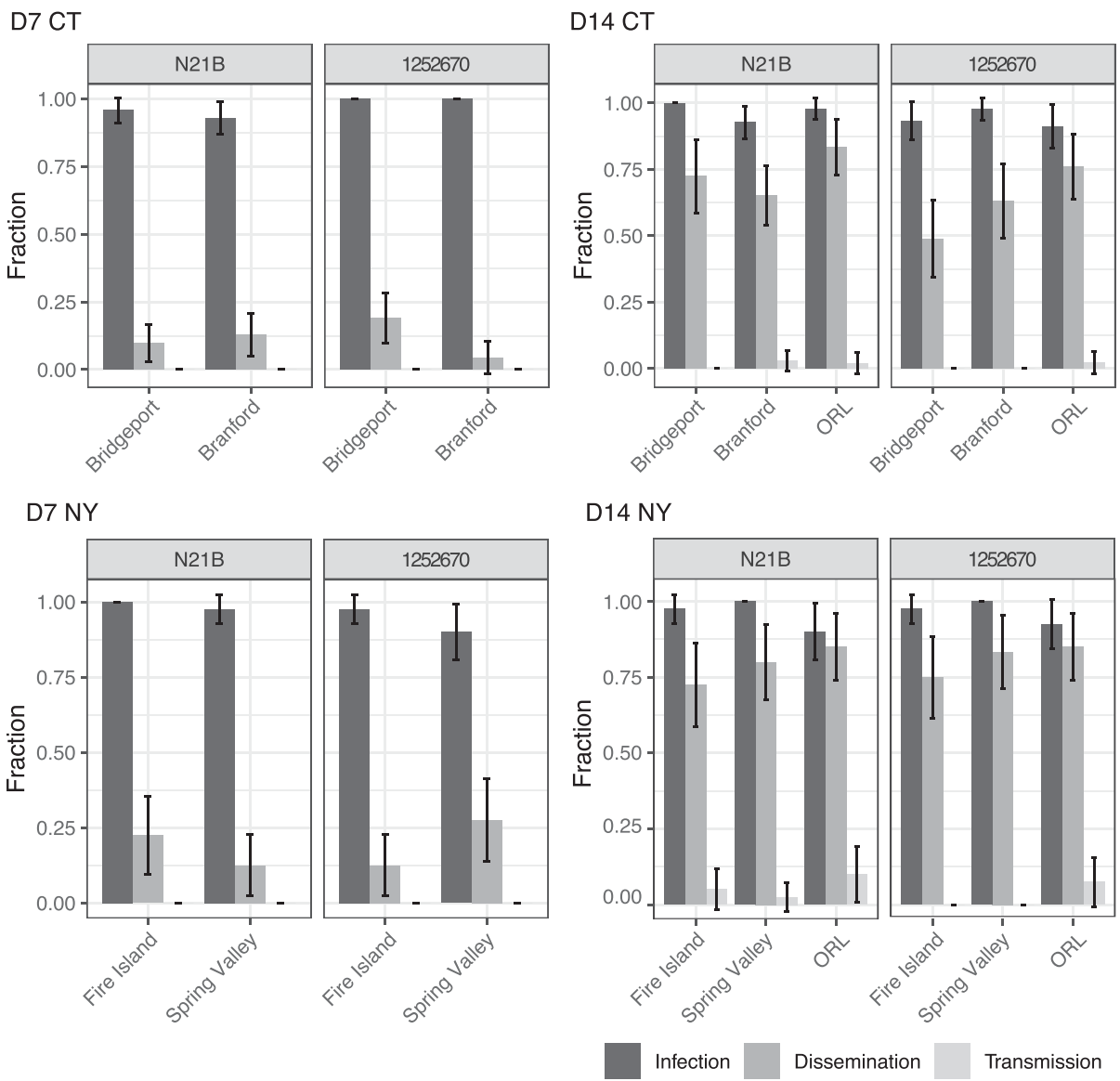

FiguRE 2. Dengue serotype 2 virus infection, dissemination, and transmission rates of Aedes albopictus populations from Connecticut (CT: Bridgeport and Branford) and New York (NY: Fire Island and Spring Valley) at days 7 (D7) and 14 pi (D14). Percentages shown are based on the total number of mosquitoes tested. The virus strain used for the challenge is indicated in the top of each panel (NI-2B and 1252670). Error bars represent $95 \% \mathrm{Cl}$. Aedes aegypti (ORL) control is shown.

Table S4; NY: $P<0.001$, Supplemental Table S5; Figure 3 and Supplemental Table S2). All populations yield at least one positive sample during the sampling period (Supplemental Table S2).

\section{DISCUSSION}

The establishment and seasonal occurrence of Ae. albopictus in the Northeastern United States pose a public health risk to the inhabitants of this region. We found that Ae. albopictus populations from Connecticut and NY were susceptible to infection by all strains of ZIKV, DENV-2, and CHIKV tested, and virus transmission was demonstrated for some of the virus-vector pairings. Although endemic transmission of these viruses does not occur in Connecticut or New York State, travel-associated cases occur annually (CDC ArboNet; www.cdc.gov) and, under the right conditions, could lead to a local outbreak.

Among all three viruses tested, special attention should be paid to CHIKV, which exhibited higher and earlier virus dissemination and transmission rates than DENV-2 and ZIKV across the mosquito populations tested. Dissemination and transmission of CHIKV peaked at $7 \mathrm{dpi}$, whereas DENV-2 and ZIKV rates continue to increase by day 14 . This early peak was also noted in Brazilian populations by Vega-Rúa et al., ${ }^{25}$ and is in line with the earlier onset in dissemination observed.
Alphaviruses such as CHIKV are generally known to have shorter extrinsic incubation periods relative to flaviviruses, such as dengue and ZIKV. ${ }^{26-29}$ The short extrinsic incubation period reported here could allow the disease to spread quickly through the immune-naive population of the Northeastern United States on arrival. This risk pattern is similar to that found in a study conducted with European populations, where competence for a strain of chikungunya, dengue serotype 1 , and Zika, was compared, and chikungunya was determined to pose the highest threat to European public health. ${ }^{33} \mathrm{Here}$, we assayed two viral strains for each of the viruses tested to account for differences in transmission efficiencies between lineages and genotypes, known to exist within mosquito vector species and populations..$^{22,34-36}$ Our data indicate that CHIKV dissemination rate is dependent on the virus strain infecting the mosquitoes and on the Ae. albopictus population. We found that the strain of CHIKV virus played a significant role in dissemination and transmission rates of CHIKV from CT and NY. The LR2006OPY1 strain led to significantly more $A e$. albopictus with disseminated infections than the IDR140025461 strain. LR2006OPY1 belongs to the Indian Ocean lineage of CHIKV that triggered the 2005-2006 major outbreak and carries an A226V substitution in the envelope protein gene (E1), known to be an adaptation for efficient transmission by Ae. albopictus. ${ }^{37,38}$ Simultaneously, we also observed that certain populations of $A e$. albopictus 


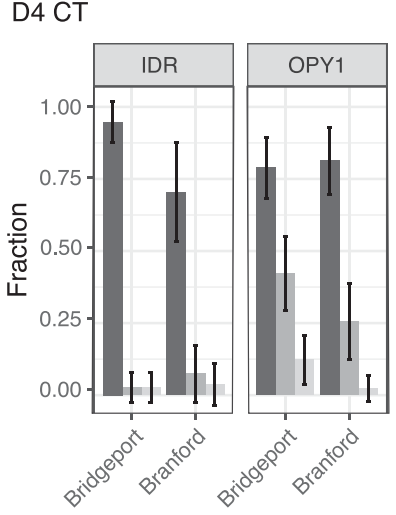

D4 NY

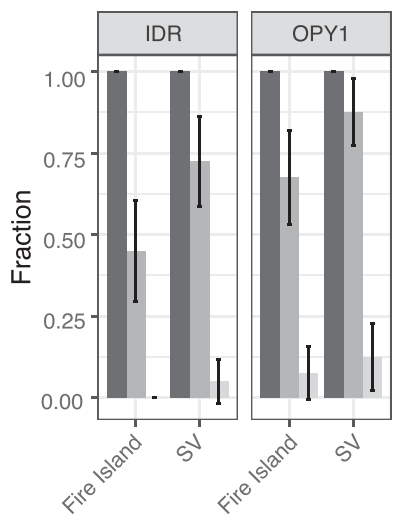

D7 CT

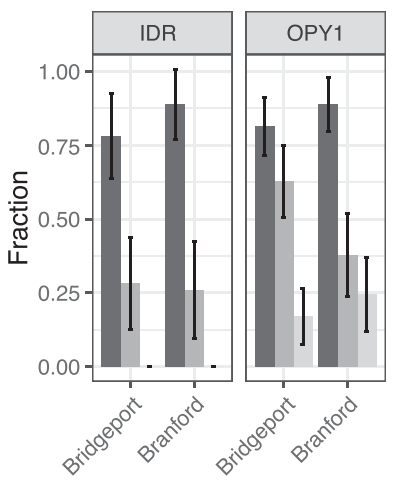

D7 NY

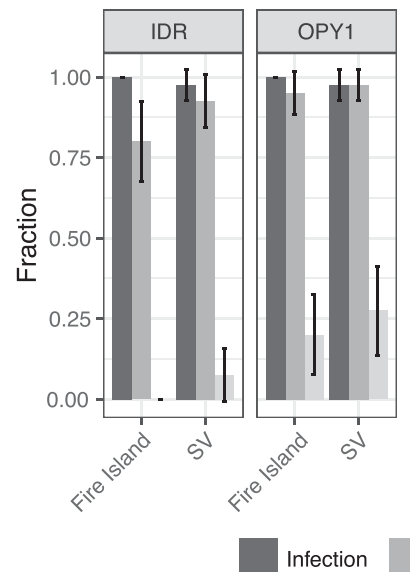

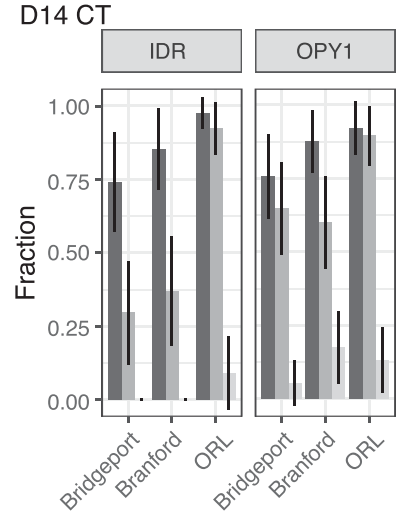

D14 NY

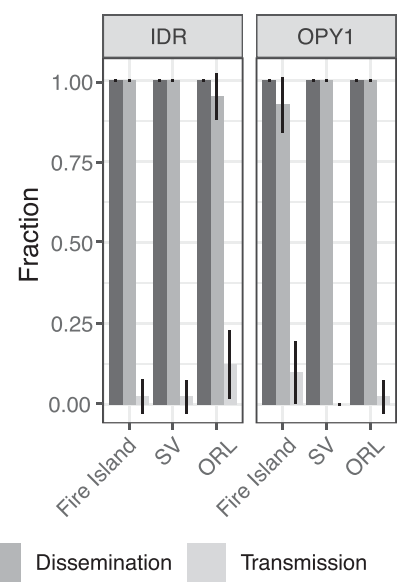

FIGURE 3. Chikungunya virus infection, dissemination, and transmission rates of Aedes albopictus populations from Connecticut (CT: Bridgeport and Branford) and New York (NY: Fire Island and Spring Valley) at days 4, 7, and 14 pi (D4, D7, and D14, respectively). Percentages shown are based on the total number of mosquitoes tested. The virus strain used for the challenge is indicated in the top of each panel (IDR: IDR140025461, OPY1: LR2006OPY1). Error bars represent 95\% Cl. Aedes aegypti (ORL) control is shown.

were more susceptible to CHIKV than others, particularly the Spring Valley population in NY which displayed consistently higher dissemination rates. The observed dissemination pattern supports previous findings indicating that the risk of a local epidemic will depend on the particular lineage of the virus imported to the region and on the local mosquito population. ${ }^{22,35,36,39}$

Our results show a very homogeneous response for DENV2 across all populations and viral strains tested, with no specific driver identified for DENV-2 competence. Based on the high infection rates observed for all treatments $(<93 \%)$, it is possible that the viral titers used may have overwhelmed any existing differences, which may become evident if we had used a lower viral dose. By contrast, ZIKV rates were highly variable and differ considerably between CT and NY, with viral strain and population background driving variability in the later. Strain-driven variability of competence for ZIKV in NY Ae. albopictus has been previously reported in Ciota et al. ${ }^{22}$ with a population originated in Suffolk County, NY. Although we have expanded those experiments to include two strains of each virus and two mosquito populations from each state, our study is by no means an exhaustive comparison, and different viral strains and mosquito populations may still play an important role in determining the risk of the Northeastern U.S. region to infection by ZIKV and DENV-2, as well as for CHIKV.
Transmission, based on the detection of virus in saliva, was overall low for all viruses, with $<2.9 \%$ for the two flaviviruses tested: ZIKV and DENV-2; and an order of magnitude higher (up to $27.5 \%$ ) for CHIKV, the only alphavirus in this study. These low transmission rates estimated from saliva samples, despite relatively high rates of dissemination across our study, are frequently reported ${ }^{40-45}$ and likely reflect limitations of the methodologies used to estimate transmission. ${ }^{46}$ All of these studies used forced salivation techniques, which do not mimic the mechanism by which mosquitoes probe or feed naturally. We are currently evaluating the ability of in vitro methods to estimate arbovirus transmission to vertebrate hosts to determine if this discrepancy is an artifact of the methodology. Details on this project are the subject of a future publication by the authors. Regardless, we should emphasize that although we report lower dissemination rates for ZIKV and DENV-2, relative to $\mathrm{CHIKV}$, the extent of dissemination for these viruses remains relevant, reaching relatively high numbers by day 14 pi (ZIKV: 15-98\%; DENV-2: 49-84\%), and should be taken into account when assessing the local risk of virus transmission by Ae. albopictus.

The present study was conducted across two state laboratories, one from Connecticut and one from New York. Comparison of vector competence studies is known to be difficult across laboratories because of their complexity, 
reflected in the wide variety of parameters that can influence results (reviewed $\mathrm{in}^{47,48}$ ). In an attempt to minimize laboratory effects, the two laboratories involved in the current study shared protocols and used the same Ae. aegypti control. Nevertheless, there were slight differences in available materials and reagents among laboratories, which may have affected the outcomes. For example, wing dissection before saliva collection is likely to induce additional stress on the mosquitoes, which in turn could impact salivation rate; alternatively, a mosquito with wings may be able to wriggle away from the capillary or pipet tip reducing the time of effective saliva collection. Differences in elution volumes could lead to changes in the sensitivity of the RT-PCR assay to detect virus; thus, appropriate controls should be implemented such as the use of a standard curve in this study. Such differences may explain the putative laboratory effect detected on the dissemination rate of ZIKV and on the infection rate of CHIKV, based on comparison of the Ae. aegypti control. Because of the laboratory effects detected in our data, we conducted all data analyses independently for the two laboratories to minimize confounding effects. These results illustrate the sensitivity of vector competence studies to external parameters and the challenge of developing robust assays to standardize them. ${ }^{48}$

The recent arrival and establishment of Ae. albopictus in Northeastern United States pose a risk for local transmission of arboviruses that cause human disease, particularly in the absence of native herd immunity to the pathogens under consideration in this study. Zika virus, DENV-2, and CHIKV are not endemic to the region; nevertheless, imported human cases are reported annually, and our work suggests that under optimal circumstances, Ae. albopictus can support localized virus transmission. These results emphasize the importance of maintaining robust mosquito surveillance programs that target $A e$. albopictus and the development of vector control programs to reduce population densities and limit further range expansion of this species.

Received July 17, 2020. Accepted for publication November 10, 2020.

Published online December 21, 2020.

Note: Supplemental tables appear at www.ajtmh.org.

Acknowledgments: We would like to thank Laura Harrington (Cornell University) for providing NYS Ae. albopictus used in these studies and E. Harris (UC Berkley) for DENV-2 strains. J. J. Shepard, T. Petruff, R. McGuire, and E. McEwen for help rearing the colonies. This publication was funded by the Cooperative Agreement U01CK000509, funded by the CDC.

Disclaimer: The contents of the manuscript are solely the responsibility of the authors and do not necessarily represent the official views of the CDC or the Department of Health and Human Services.

Authors' addresses: Andrea Gloria-Soria and Philip M. Armstrong, Department of Environmental Sciences, Center for Vector Biology and Zoonotic Diseases, The Connecticut Agricultural Experiment Station, New Haven, CT, E-mails: andrea.gloria-soria@ct.gov, and philip.armstrong@ct.gov. Anne F. Payne, Sean M. Bialosuknia, Jessica Stout, and Nicholas Mathias, The Arbovirus Laboratories, Wadsworth Center, New York State Department of Health, Slingerlands, NY, E-mails: anne.payne@health.ny.gov, sean.bialosuknia@ health.ny.gov, jessica.stout@health.ny.gov, and nicholas.mathias@ health.ny.gov. Gillian Eastwood, Department of Environmental Sciences, Center for Vector Biology and Zoonotic Diseases, The Connecticut Agricultural Experiment Station, New Haven, CT, and Department of Entomology, Virginia Polytechnic Institute and State University, Blacksburg, VA, E-mail: geastwood@vt.edu. Alexander
T. Ciota and Laura D. Kramer, The Arbovirus Laboratories, Wadsworth Center, New York State Department of Health, Slingerlands, CT, and Department of Biomedical Sciences, State University of New York at Albany School of Public Health, Albany, NY, E-mails: alexander.ciota@health.ny.gov and laura.kramer@health.ny.gov.

This is an open-access article distributed under the terms of the Creative Commons Attribution (CC-BY) License, which permits unrestricted use, distribution, and reproduction in any medium, provided the original author and source are credited.

\section{REFERENCES}

1. Bonizzoni M, Gasperi G, Chen X, James AA, 2013. The invasive mosquito species Aedes albopictus: current knowledge and future perspectives. Trends Parasitol 29: 460-468.

2. Moore CG, Mitchell CJ, 1997. Aedes albopictus in the United States: ten-year presence and public health implications. Emerging Infect Dis 3: 329-334.

3. Hahn MB, Eisen RJ, Eisen L, Boegler KA, Moore CG, McAllister J, Savage HM, Mutebi JP, 2016. Reported distribution of Aedes (Stegomyia) aegypti and Aedes (Stegomyia) albopictus in the United States, 1995-2016 (Diptera: Culicidae). J Med Entomol 53: 1169-1175.

4. Kache PA, Eastwood G, Collins-Palmer K, Katz M, Falco RC, Bajwa WI, Armstrong PM, Andreadis TG, Diuk-Wasser MA, 2020. Environmental determinants of Aedes albopictus abundance at a northern limit of its range in the United States. Am J Trop Med Hyg 102: 436-447.

5. Faraji A, Egizi A, Fonseca DM, Unlu I, Crepeau T, Healy SP, Gaugler R, 2014. Comparative host feeding patterns of the asian tiger mosquito, Aedes albopictus, in urban and suburban northeastern USA and implications for disease transmission. PLoS Negl Trop Dis 8: e3037.

6. Muñoz J, Eritja R, Alcaide M, Montalvo T, Soriguer RC, Figuerola J, 2011. Host-feeding patterns of native Culex pipiens and invasive Aedes albopictus mosquitoes (Diptera: Culicidae) in urban zones from Barcelona, Spain. J Med Entomol 48: 956-960.

7. Gratz NG, 2004. Critical review of the vector status of Aedes albopictus. Med Vet Entomol 18: 215-227.

8. Paupy C, Delatte H, Bagny L, Corbel V, Fontenille D, 2009. Aedes albopictus, an arbovirus vector: from the darkness to the light. Microbes Infect 11: 1177-1185.

9. Reiter P, Fontenille D, Paupy C, 2006. Aedes albopictus as an epidemic vector of chikungunya virus: another emerging problem? Lancet Infect Dis (Print). 6: 463-464.

10. Rezza G et al., 2007. Infection with chikungunya virus in Italy: an outbreak in a temperate region. Lancet 370: 1840-1846.

11. Lindh $\mathrm{E}$ et al., 2019. The Italian 2017 outbreak chikungunya virus belongs to an emerging Aedes albopictus-adapted virus cluster introduced from the Indian subcontinent. Open Forum Infect Dis 6: ofy32.

12. Effler PV et al., 2005. Dengue fever, Hawaii, 2001-2002. Emerg Infect Dis 11: 742-749.

13. Hasty JM et al., 2020. Entomological investigation detects dengue virus Type 1 in Aedes (Stegomyia) albopictus (Skuse) during the 2015-16 outbreak in Hawaii. Am J Trop Med Hyg 102: 869-875.

14. Luo L, Liang H, Hu Y, Liu W, Wang Y, Jing Q, Zheng X, Yang Z, 2012. Epidemiological, virological, and entomological characteristics of dengue from 1978 to 2009 in Guangzhou, China. $J$ Vector Ecol 37: 230-240.

15. Tsuda $Y$, Maekawa $Y$, Ogawa K, Itokawa K, Komagata O, Sasaki $\mathrm{T}$, Isawa H, Tomita T, Sawabe K, 2015. Biting density and distribution of Aedes albopictus during the September 2014 outbreak of dengue fever in Yoyogi Park and the vicinity in Tokyo Metropolis, Japan. Jpn J Infect Dis 69: 1-5.

16. Weaver SC, Reisen WK, 2010. Present and future arboviral threats. Antiviral Res 85: 328-345.

17. Musso D, Cao-Lormeau VM, Gubler DJ, 2016. Zika virus: following the path of dengue and chikungunya? Lancet 386 : 243-244.

18. Lwande OW, Obanda V, Lindström A, Ahlm C, Evander M, Näslund J, Bucht G, 2019. Globe-trotting Aedes aegypti and 
Aedes albopictus: risk factors for arbovirus pandemics. VectorBorne Zoonotic Dis 20: 71-81.

19. Armstrong PM, Andreadis TG, Shepard JJ, Thomas MC, 2017. Northern range expansion of the Asian tiger mosquito (Aedes albopictus): analysis of mosquito data from Connecticut, USA. PLoS Negl Trop Dis 11: e0005623.

20. Nelson B, Morrison S, Joseph H, Wojno A, Lash RR, Haber Y, Berro A, Cetron M, Grills A, 2016. Travel volume to the United States from countries and U.S. territories with local Zika virus transmission. PLoS Curr 8: ecurrents. outbreaks. ac6d0f8c9c35e88825c1a1147697531c.

21. Roundy CM et al., 2017. Variation in Aedes aegypti mosquito competence for Zika virus transmission. Emerg Infect Dis 23: 625-632.

22. Ciota AT, Bialosuknia SM, Zink SD, Brecher M, Ehrbar DJ, Morrissette MN, Kramer LD, 2017. Effects of Zika virus strain and Aedes mosquito species on vector competence. Emerg Infect Dis 23: 1110-1117.

23. Gloria-Soria A, Soghigian J, Kellner D, Powell JR, 2019. Genetic diversity of laboratory strains and implications for research: the case of Aedes aegypti. PLoS Negl Trop Dis 13: e0007930.

24. Payne AF, Binduga-Gajewska I, Kauffman EB, Kramer LD, 2006. Quantitation of flaviviruses by fluorescent focus assay. $J$ Virol Methods 134: 183-189.

25. Vega-Rúa A, Zouache K, Girod R, Failloux AB, Lourenço-deOliveira R, 2014. High level of vector competence of Aedes aegypti and Aedes albopictus from ten American countries as a crucial factor in the spread of chikungunya virus. $J$ Virol 88: 6294-6306.

26. Amraoui F, Ben Ayed W, Madec Y, Faraj C, Himmi O, Btissam A, Sarih M, Failloux AB, 2019. Potential of Aedes albopictus to cause the emergence of arboviruses in Morocco. PLoS Negl Trop Dis 13: e0006997.

27. Robison A, Young MC, Byas AD, Rückert C, Ebel GD, 2020. Comparison of chikungunya virus and Zika virus replication and transmission dynamics in Aedes aegypti mosquitoes. Am J Trop Med Hyg 103: 869-875.

28. Dubrulle M, Mousson L, Moutailler S, Vazeille M, Failloux AB, 2009. Chikungunya virus and Aedes mosquitoes: saliva is infectious as soon as two days after oral infection. PLoS One 4: e5895.

29. Miller LK, Ball LA, 2012. The Insect Viruses. New York: Springer Science \& Business Media.

30. R Core Team, 2017. R: A Language and Environment for Statistical Computing. Vienna, Austria: R Foundation for Statistical Computing. Available at: https://www.R-project.org/.

31. Bates D, Mächler M, Bolker B, Walker S, 2014. Fitting Linear Mixed-Effects Models using Ime4. arXiv:14065823 [stat]. Available at: http://arxiv.org/abs/1406.5823. Accessed July 8, 2020.

32. Fox J, Weisberg S, 2011. An R Companion to Applied Regression. Sage Publications.

33. Mariconti M, Obadia T, Mousson L, Malacrida A, Gasperi G, Failloux $A B$, Yen PS, 2019. Estimating the risk of arbovirus transmission in Southern Europe using vector competence data. Sci Rep 9: 17852.

34. Vega-Rua A, Zouache K, Caro V, Diancourt L, Delaunay P, Grandadam M, Failloux AB, 2013. High efficiency of temperate Aedes albopictus to transmit chikungunya and dengue viruses in the Southeast of France. PLoS One 8: e59716.

35. Zouache K, Fontaine A, Vega-Rua A, Mousson L, Thiberge JM, Lourenco-De-Oliveira R, Caro V, Lambrechts L, Failloux AB, 2014. Three-way interactions between mosquito population, viral strain and temperature underlying chikungunya virus transmission potential. Proc $R$ Soc 281: 20141078.

36. Gloria-Soria A, Armstrong PM, Powell JR, Turner PE, 2017. Infection rate of Aedes aegypti mosquitoes with dengue virus depends on the interaction between temperature and mosquito genotype. Proc Biol Sci 284: 20171506.

37. Schuffenecker I et al., 2006. Genome microevolution of chikungunya viruses causing the Indian ocean outbreak. PLOS Med 3: e263.

38. Tsetsarkin KA, Vanlandingham DL, McGee CE, Higgs S, 2007. A Single mutation in chikungunya virus affects vector specificity and epidemic potential. PLoS Pathog 3: e201.

39. Lambrechts L, Chevillon C, Albright RG, Thaisomboonsuk B, Richardson JH, Jarman RG, Scott TW, 2009. Genetic specificity and potential for local adaptation between dengue viruses and mosquito vectors. BMC Evol Biol 9: 160.

40. Richards SL, Anderson SL, Alto BW, 2012. Vector competence of Aedes aegypti and Aedes albopictus (Diptera: Culicidae) for dengue virus in the Florida keys. J Med Entomol 49: 942-946.

41. Diagne CT et al., 2015. Potential of selected Senegalese Aedes spp. mosquitoes (Diptera: Culicidae) to transmit Zika virus. BMC Infect Dis 15: 492.

42. Ryckebusch F, Berthet M, Missé D, Choumet V, 2017. Infection of a French population of Aedes albopictus and of Aedes aegypti (paea strain) with Zika virus reveals low transmission rates to these vectors' saliva. Int J Mol Sci 18: 2384.

43. Garcia-Luna SM et al., 2018. Variation in competence for ZIKV transmission by Aedes aegypti and Aedes albopictus in Mexico. PLoS Negl Trop Dis 12: e0006599.

44. Hernández-Triana LM et al., 2019. Evidence for infection but not transmission of Zika virus by Aedes albopictus (Diptera: Culicidae) from Spain. Parasit Vectors 12: 204.

45. Gaye A, Wang E, Vasilakis N, Guzman H, Diallo D, Talla C, Ba Y, Dia I, Weaver SC, Diallo M, 2019. Potential for sylvatic and urban Aedes mosquitoes from Senegal to transmit the new emerging dengue serotypes 1, 3 and 4 in West Africa. PLoS Negl Trop Dis 13: e0007043.

46. Smith DR, Carrara AS, Aguilar PV, Weaver SC, 2005. Evaluation of methods to assess transmission potential of venezuelan equine encephalitis virus by mosquitoes and estimation of mosquito saliva titers. Am J Trop Med Hyg 73: 33-39.

47. Souza-Neto JA, Powell JR, Bonizzoni M, 2019. Aedes aegypti vector competence studies: a review. Infect Genet Evol 67: 191-209.

48. Azar SR, Weaver SC, 2019. Vector competence: what has Zika virus taught us? Viruses 11: 867. 